\title{
Metamaterial CRLH Antennas on Silicon Substrate for Millimeter-Wave Integrated Circuits
}

\author{
Gheorghe Ioan Sajin ${ }^{1}$ and Iulia Andreea Mocanu ${ }^{1,2}$ \\ ${ }^{1}$ National Institute for Research and Development in Microtechnologies (IMT-Bucharest) Erou Iancu Nicolae 126A, \\ 077190 Bucharest, Romania \\ ${ }^{2}$ Faculty of Electronics, Telecommunications and Informations Engineering, Politehnica University Bucharest, \\ Boulevard Iuliu Maniu 1-3, 061071 Bucharest, Romania \\ Correspondence should be addressed to Gheorghe Ioan Sajin, gheorghe.sajin@imt.ro \\ Received 5 September 2012; Accepted 4 November 2012 \\ Academic Editor: James R. Kelly
}

Copyright (๑) 2012 G. I. Sajin and I. A. Mocanu. This is an open access article distributed under the Creative Commons Attribution License, which permits unrestricted use, distribution, and reproduction in any medium, provided the original work is properly cited.

The paper presents two composite right/left-handed (CRLH) coplanar waveguide (CPW) zeroth-order resonant (ZOR) antennas which were designed, processed, and electrically characterized for applications in the millimetric wave frequency range. Two CRLH antennas were developed for $f=27 \mathrm{GHz}$ and $f=38.5, \mathrm{GHz}$, respectively. The CRLH antenna on $f=27 \mathrm{GHz}$ shows a return loss of $\mathrm{RL}<-18.78 \mathrm{~dB}$ at $f=26.88 \mathrm{GHz}$. The $-3 \mathrm{~dB}$ radiation characteristic beamwidth was approximately $37^{\circ}$ and the gain was $G_{i}=2.82 \mathrm{dBi}$. The CRLH antenna on $f=38.5 \mathrm{GHz}$ has a return loss of $\mathrm{RL}<-38.5 \mathrm{~dB}$ at $f=38.82 \mathrm{GHz}$ and the $-3 \mathrm{~dB}$ radiation characteristic beamwidth of approximately $17^{\circ}$. The gains were $G_{i}=1.08 \mathrm{dBi}$ at $f=38 \mathrm{GHz}$ and $G_{i}=1.2 \mathrm{dBi}$ at $f=38.6 \mathrm{GHz}$. The maximum measured gain was $G_{i}=1.75 \mathrm{dBi}$ at $f=38.2 \mathrm{GHz}$. It is, upon the authors' knowledge, the first report of millimeter wave CRLH antennas on silicon substrate in CPW technique for use in mm-wave monolithic integrated circuit.

\section{Introduction}

In recent years the area of metamaterials has been getting a lot of attention from the scientific community. Although Veselago enunciated the theory of left-handed (LH) materials more than 50 years ago [1], structures mimicking these properties were developed only about 10 years ago [2].

Considering the transmission line (TL) parameters, metamaterials were introduced as the concept of Composite Right/Left-Handed (CRLH-TL). The CRLH is an artificial TL that can be obtained by combining the RH behavior of the classical TL modeled by a transmission line loaded with series connected inductors and parallel grounded capacitors, with the LH behavior modeled by series connected capacitors and parallel connected grounded inductors. Such a transmission line exhibits both right-handed (RH) and left-handed (LH) behavior. The first presentation of this type of transmission line and of devices made on this basis is done in [3].
This particular frequency characteristic of the CRLH TL has been exploited in the development of many types of devices such as coupled-line directional couplers, filters and resonators and various types of antennas [4-14].

A complete description of the most practical leaky wave and ZOR antennas was done in [4].

The current trend in electronics shows that there is a need of devices with reduced size planar topology and easily integrable with active components in microwave and $\mathrm{mm}$-wave integrated circuits. Although hundreds of antennas configurations on metamaterials have been reported in literature, almost all are demonstrators processed on soft materials for frequencies up to $10 \mathrm{GHz}-12 \mathrm{GHz}$. Very few antennas have been manufactured on semiconductor materials, $[6-8,15,16]$, in the mm-wave frequency range, $[8,15-17]$, and ever the less have been built for use in practical microwave and millimeter wave integrated circuits [17-21]. In the quest of more efficiency when allocating the 
frequency bandwidths, countries like Australia and Canada have chosen to use the $27 \mathrm{GHz}$ spectrum and $38 \mathrm{GHz}$ spectrum, respectively, for broadband wireless services and for a wide variety of fixed service applications. So, there is the need for using antennas designed to work on this frequencies.

CRLH antennas presented in this paper were processed in coplanar waveguide (CPW) configuration on silicon substrate and have been designed to be used in practical applications. The CPW technology was preferred due to an easier technological approach and also due to a straightforward measurement procedure using an on-wafer characterization installation. It is, upon the authors' knowledge, the first realization of a millimeter wave CRLH antennas on silicon substrate for effective use in mm-wave integrated circuits.

\section{CRLH Antenna Structure}

The devices cf. $[15,16]$ designed in this paper are zerothorder resonance (ZOR) antennas consisting of an openended array of CPW CRLH transmission lines cells. Each cell has a $\mathrm{T}$ circuit topology made of two series connected CPW interdigital capacitors and two parallel conected short-ended $\mathrm{CPW}$ transmission lines as inductive stubs. Unlike microstrip antenna, using CPW transmission lines allows obtaining much smaller circuit area because no large patch area and no vias to the ground are needed. The elementary CRLH cell used in antenna construction is depicted in Figure 1(a) while in Figure 1(b) equivalent circuit of the CRLH cell is presented.

Here $2 C_{L}$ and $L_{R} / 2$ are the equivalent capacitance and the equivalent inductance of the series capacitor, while $L_{L}$ is the equivalent parallel inductance of the two CPW inductive stubs and $C_{R}$ is the equivalent parallel capacitance. The parallel capacitance $C_{R}$ includes, also, the equivalent parallel capacitance to the ground of the interdigital capacitors. It is important to point out that the $L_{R} / 2$ and $C_{R}$ are strongly related to $2 C_{L}$ and $L_{L}$ values. A similar CRLH topology was presented for a leaky-wave antenna in [4].

The design was made for obtaining a balanced CRLH structure for which $1 / \sqrt{L_{L} C_{R}}=1 / \sqrt{L_{R} C_{L}}$. The starting point in designing the capacitor is to choose: (i) the operating resonance frequency $f_{\mathrm{sh}}=1 / 2 \pi \sqrt{L_{L} C_{R}}$; (ii) the length of the capacitor fingers so that the overall length of the CRLH cell would be much smaller comparing to the operating wavelength; (iii) the geometry of the interdigital capacitor allowed by the technological limitations.

\section{Antenna Design and Processing on Silicon Substrate}

Two devices working at the frequencies $f=27 \mathrm{GHz}$ and $38.5 \mathrm{GHz}$, respectively, were designed, technologically processed, and electrically characterized. In the following, antenna at $27 \mathrm{GHz}$ will be termed as Antenna \#1 and antenna at $38.5 \mathrm{GHz}$ will be termed as Antenna \#2.

3.1. $27 \mathrm{GHz}$ Antenna Design. A CPW CRLH ZOR antenna at the frequency $f=27 \mathrm{GHz}$ was designed, technologically processed, and electrically measured. It is considered to be used in broadband wireless systems for the $27 \mathrm{GHz}$ spectrum in Australia. The device was made of three resonant CRLH cells made on a $5 \mathrm{k} \Omega \mathrm{cm}$ silicon wafer. The values of the elements of the CRLH equivalent circuit have been computed for $Z_{C}=50 \Omega$.

The obtained values for CRLH elementary cell components (see Figure 1(b)) are: $L_{R}=0.6 \mathrm{nH}, L_{L}=0.13 \mathrm{nH}$, $C_{R}=267 \mathrm{fF}$, and $C_{L}=26 \mathrm{fF}$. For these values the computed resonant frequency is $f_{\mathrm{sh}}=27 \mathrm{GHz}$.

In order to obtain the antenna radiating structure in $\mathrm{CPW}$ configuration, the following geometrical dimensions of CRLH cell layout (capacitors, inductors, and cell dimension) are obtained (see Figure 1(a)):

(i) for the inductive CPW stub: $l_{L}=212 \mu \mathrm{m} ; w_{L}=$ $42 \mu \mathrm{m} ; s_{L}=10 \mu \mathrm{m}$;

(ii) for the interdigital capacitors: $w_{C}=10 \mu \mathrm{m} ; s_{C}=$ $5 \mu \mathrm{m} ; l_{C}=250 \mu \mathrm{m}$ and $g_{C}=65 \mu \mathrm{m}$; the number of digits was 10 .

The length of the CRLH cell is $p=690 \mu \mathrm{m}$ fulfilling the condition $p \ll \lambda_{g}$ where $\lambda_{g}=4370 \mu \mathrm{m}$ is the wavelength at $f=27 \mathrm{GHz}$. The surface occupied by the radiant antenna structure composed of 3 CLRH cells is approx. $2 \mathrm{~mm} \times 1.2 \mathrm{~mm} \cong 2.4 \mathrm{~mm}^{2}$. To compare, a patch microstrip antenna processed on silicon using the same technology, working on the fundamental mode at the same frequency has a surface of approx. $2.2 \mathrm{~mm} \times 1.44 \mathrm{~mm} \cong 3.17 \mathrm{~mm}^{2}$. One may see that the CRLH antenna allows a surface size reduction of approximately $25 \%$ that is very important in the management of the mm-wave circuit planar dimensions.

The antenna input is made of a feeding line of $3400 \mu \mathrm{m}$ length and the geometry computed to match the $50 \Omega$ characterisctic impedance of the mm-wave circuit. This geometry allows antenna structure mounting on a dedicated test fixture for radiation characteristic and gain measurement.

3.2. 38.5 GHz Antenna Design. Using the same design conditions as for the previous described antenna, a CPW CRLH ZOR antenna on the frequency $f=38.5 \mathrm{GHz}$ was designed, processed, and measured. This type of antenna is considered to be used in the point to point systems in the $38 \mathrm{GHz}$ spectrum in Canada. The elementary CRLH CPW cell has the same layout as it was presented in Figure 1. The antenna is formed also of three resonant CRLH cells processed on the same kind of silicon wafer. The computed values of interdigital capacitors and the inductive CPW lines for a frequency $f=38.5 \mathrm{GHz}$ are the following: $L_{R}=0.4 \mathrm{nH}, L_{L}=$ $0.08 \mathrm{nH}, C_{R}=210 \mathrm{fF}$, and $C_{L}=18 \mathrm{fF}$. For these values the resonant frequency computed with (1) give $f_{\text {sh }}=38.8 \mathrm{GHz}$.

The physical dimensions necessary to obtain a CRLH cell with the previous capacitor and inductor values are the following (see Figure 1(a)):

(i) for the inductive CPW stub: $l_{L}=212 \mu \mathrm{m} ; w_{L}=$ $40 \mu \mathrm{m} ; s_{L}=8 \mu \mathrm{m}$;

(ii) for the interdigital capacitor: $l_{C}=250 \mu \mathrm{m}, w_{C}=$ $7 \mu \mathrm{m}, s_{C}=8 \mu \mathrm{m}, g_{C}=65 \mu \mathrm{m}$ and number of digits: 10. 




(a)



(b)

Figure 1: CPW CRLH elementary cell used in antenna construction (a) and the equivalent circuit (b).

Also at this frequency, the length of the CRLH cell is well under the wavelength fulfilling the condition $p=690 \mu \mathrm{m}$ $\ll \lambda_{g}=3110 \mu \mathrm{m}$.

The antennas were processed on a $400 \mu \mathrm{m}$ thick silicon wafer $\left(\varepsilon_{r} \cdot \mathrm{Si}=11.9\right)$ with a resistivity of $5 \mathrm{k} \Omega \mathrm{cm}$. On the silicon surface was grown through thermal oxidation a $1 \mu \mathrm{m}$ thick $\mathrm{SiO}_{2}$ layer $\left(\varepsilon_{r} \cdot \mathrm{SiO}_{2}=3.9\right)$. The metallization was obtained by evaporation of $4.000 \AA \mathrm{Au} / 500 \AA \mathrm{Cr}$. By using silicon, the possibility of integration with active devices in a functional circuit is left open.

Two technological processing methods were tried: (i) standard wet etching photolithography and (ii) laser ablation.

3.3. Technological Approach. Standard photolithography as technology for antenna processing consists of photoresist deposition, masking, exposure, developing, wet etching; photoresist remains removal.

A quarter of a silicon wafer supporting some CRLH antenna structures with a Süss Microtec on-wafer $67 \mathrm{GHz}$ probe-tip contacting an antenna feeding line in order to measure the resonant frequency and return loss is presented in Figure 2(a). In Figure 2(b), the layout of a CRLH cell interdigital capacitors and inductor lines is presented.

Concerning photolithography, this technology is able to provide excellent layouts for microwave devices. A drawback is the tendency of metallic lines over-etching. During our experiments there was necessary to apply a correction factor in the device mask design in order to keep the ratio $10 \mu \mathrm{m} / 5 \mu \mathrm{m}$ between metallic finger width and space between the metallic fingers

In order to obtain devices by laser ablation, a twostep technological process was applied. The first step was the removal by a standard photolithographic process of the metal from the large areas, such as the gaps between the signal line and the ground planes. For this, a mask without the interdigital capacitors was designed. The second step consisted of tracing the fine details of the interdigital capacitors gaps by laser ablation.

A direct laser writing (DLW) method was implemented to microprocess the $\mathrm{Au} / \mathrm{Cr}$ layers evaporated on silicon. The samples were laser ablated by tightly focusing a femtosecond laser with $200 \mathrm{fs}$ pulse duration, $775 \mathrm{~nm}$ wavelength, tens of nanojoules pulse energy, and $2 \mathrm{kHz}$ repetition rate. The $2 \mathrm{D}$ structures were generated according to a computer controlled algorithm by precisely translating the sample with resolution below $1 \mu \mathrm{m}$.

Figure 3(a) shows the antenna layout after photolithography in the first step. There can be observed the grounded lines forming the inductive stubs and the areas where the interdigital capacitors are to be processed through laser ablation. Figure 3(b) shows the same area after the capacitors were processed through the laser ablation method. A good definition of the lines could be observed: the lines were straight and the rounding at the corners was minimal. The geometry was accurately kept.

By applying these two steps process, we can benefit from the fast and cost-effective standard photolithographic process which maintains good accuracy for larger geometries (tens or hundreds of microns) and the highly accurate but time consuming laser ablation process in order to obtain fine details (micronic and submicronic geometries).

However, this technology was not retained due to the following drawback: during the laser ablation a cloud of removed metallic material is spread all over the interdigital capacitor structure, including the space between the 


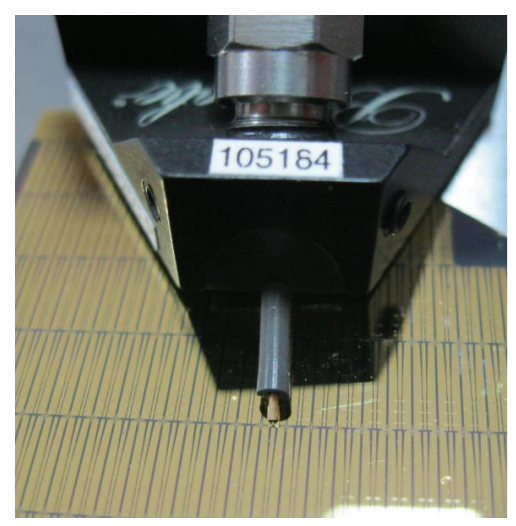

(a)

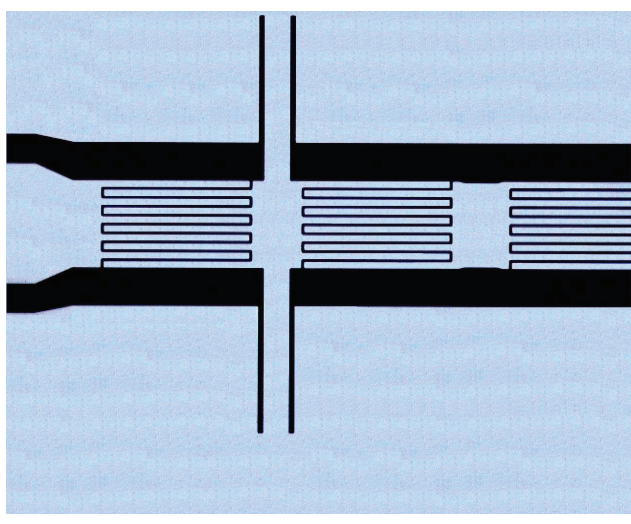

(b)

Figure 2: Silicon wafer with antenna structures (a) and optical microscopy photo showing one of the CRLH cell (b).



(a)

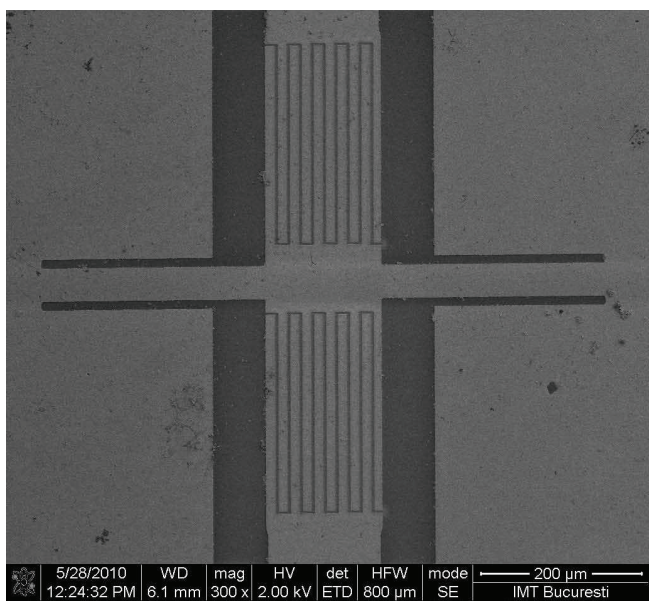

(b)

FIgURE 3: The CRLH antenna after the first step (a) and a SEM image of the CRLH cell after the second step of the laser ablation technology (b).

two digits. These metallic particles may be observed in Figure 3(b) as well as white dots in SEM image in Figure 4. This contamination dramatically increases the device's losses.

After obtaining the antenna structures and on wafer measurement of the resonant frequency and return loss, the silicon wafer was diced with an abrasive diamond wheel tool, thus obtaining separate antenna chips. These discrete structures were mounted on dedicated test fixtures in order to measure antenna's directivity characteristic and gain. Two such diced CRLH antennas are presented in Figure 5(a) while in Figure 5(b) two structures mounted on the test fixtures can be seen.

It should be noted that antennas are used in applications requiring absence of metallic layer on the back of the silicon wafer. At the same time, mounting the silicon wafer on the Süss Microtec working chuck as well as mounting the antenna structures on the test fixtures may excite the CPWG mode instead of CPW, due to the presence of metal underneath the antenna acting as ground plane. The line impedance in the CPW mode is $47.12 \Omega$ while the line impedance in the CPWG mode is $44.85 \Omega$. This difference of $2.27 \Omega$ does not dramatically affect the device measurement's results.

\section{Measurements and Experimental Results}

4.1. Measurement Techniques. As electrical characteristics of $\mathrm{CRLH}$ antenna, the return loss, the resonant frequency, the radiation characteristic, and the gain were measured.

Return loss and the resonant frequency were measured on-wafer using a 37397D vector network analyzer from Anritsu, equipped with PM5 set-up from Süss Microtec. In Figure 2(a) it can be seen a Süss Microtec on-wafer $67 \mathrm{GHz}$ probe-tip contacting a CRLH antenna feeding line in order to measure the resonant frequency and return loss.

The radiation characteristics were measured with a frequency generator Agilent E8257D and a spectrum analyzer Anritsu MS2668SC, both having full capabilities in the CRLH antennas frequency range. The measuring planes were 


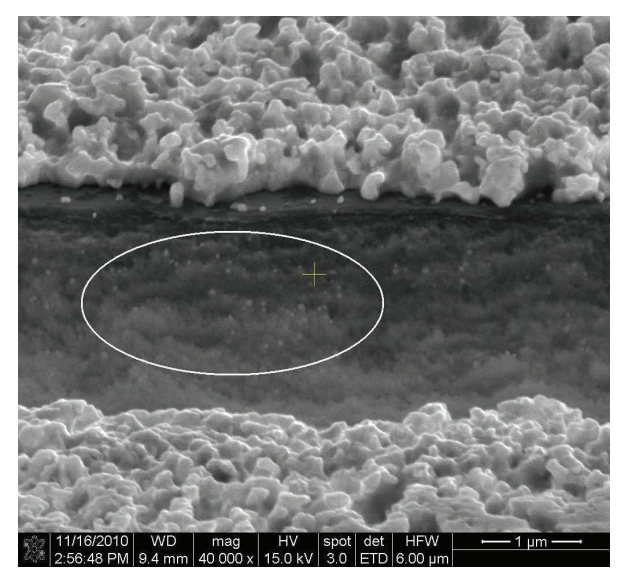

Figure 4: Technological drawbacks: metallic particles spread in the ablated area.



(a)

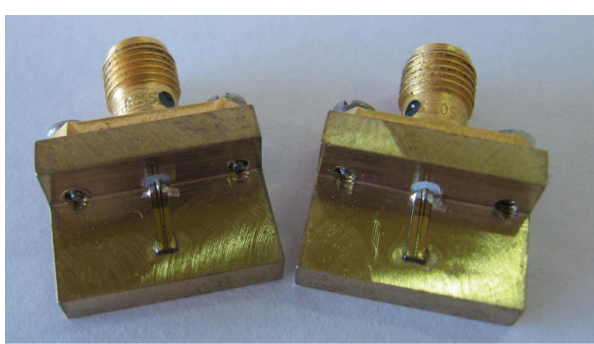

(b)

FIGURE 5: Discrete CRLH antenna structures after cutting (a) and after the mounting on a specialized test fixture (b).

the transversal plane $(\theta)$ and the longitudinal plane $(\varphi)$ as they are defined in Figure 6.

The measuring setup comprises, also, an antenna holder as is shown in Figure 7 having the possibility to rotate between $-90^{\circ}$ and $+90^{\circ}$ in transverse $(\theta)$ and longitudinal $(\varphi)$ planes. The emitting device was the CRLH antenna and as receiving device were used two frequency appropriate horn antennas connected to the spectrum analyzer. The distance between the emitting CRLH antenna and the aperture plane of the receiving horn antenna was $100 \mathrm{~mm}$. The measured power at reception was averaged over 50 measurements.

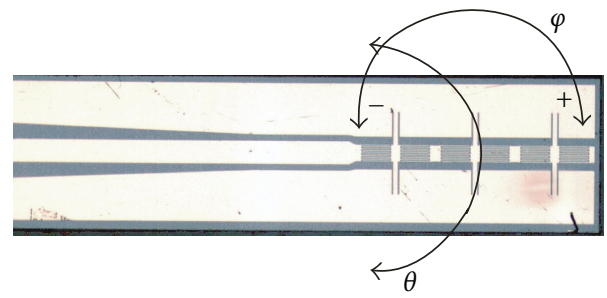

FIguRE 6: The transverse $(\theta)$ and the longitudinal $(\varphi)$ measurement planes.

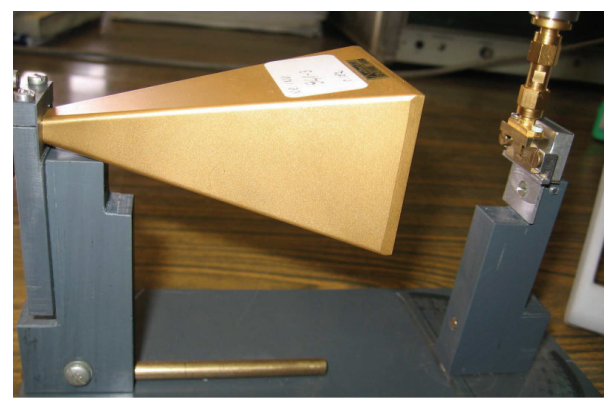

FIGURE 7: Measuring setup for CRLH antenna: detail of the measuring arrangement.

The antenna gain was obtained using the De Friis relation for two identical antennas (1):

$$
\frac{P_{r}}{P_{t}}=G_{i}^{2}\left(\frac{\lambda}{4 \pi R}\right)^{2}
$$

where $P_{t}=$ power transmitted by emitting antenna, $P_{r}=$ power at the receiving antenna, $G_{i}=$ antenna gain with respect to isotropic; $\lambda=$ wavelength, $R=$ distance between emitting and receiving antenna. In this situation the emitting and the receiving antennas are identical so that the gain of both devices are the same, $G_{i}$. The gain in ( $\mathrm{dBi}$ ) is expressed by:

$$
G(\mathrm{dBi})=10 \log _{10}\left(\frac{4 \pi R}{\lambda} \sqrt{\frac{P_{r}}{P_{t}}}\right) .
$$

The gain measurement setup shown in Figure 8 uses the same instruments utilized for the previous characterization, the arrangement of antennas holders being adapted for using the De Friis relation (2).

4.2. Experimental Results for $27 \mathrm{GHz}$ CRLH Antenna. The simulation of reflection loss of Antenna \#1 which was made with the IE3D Zeland software in the $24 \mathrm{GHz}-30 \mathrm{GHz}$ frequency band on the previously obtained geometry is presented in Figure 9. The simulation results indicate a return loss of $S_{11}=-17.94 \mathrm{~dB}$ at the frequency $f=$ $27.1 \mathrm{GHz}$.

The measured results for resonance frequency and return loss of CRLH Antenna \#1 sample are presented in Figure 10.

From Figure 10 it may be seen that the return loss and the resonant frequencies are $S_{11}=-18.78 \mathrm{~dB}$ at $f=26.88 \mathrm{GHz}$. 




FIGURE 8: Measuring setup for CRLH antenna gain.



Figure 9: Simulation of return loss of the CRLH Antenna \#1 in the 24 and $30 \mathrm{GHz}$ frequency domain.

The frequency is slightly lower than the simulated one and the return loss is smaller as well. Also, the antenna bandwidth is larger than simulated.

Radiation characteristic in transversal plane $(\theta)$ of the $27 \mathrm{GHz} \mathrm{CRLH}$ antenna is shown in Figure 11 where the received power at different angles was normalized to the maximum received power value. From Figure 11 one may see that the $-3 \mathrm{~dB}$ beam width of the radiation characteristic is between approximately $+21^{\circ}$ and $-16^{\circ}$ for meaning beam width of $37^{\circ}$.

The secondary radiation lobes have amplitudes lower than 0.3 of the main radiation lobe maximum value and occurs at the angles of approximately $\pm 40^{\circ}$.

In order to complete the characterization of the antenna's radiating capability, the radiation characteristic in longitudinal plane $(\varphi)$ was, also, measured. The results are shown in Figure 12 where the radiated power at different angles in the $(\varphi)$ plane between $-70^{\circ}$ and $+90^{\circ}$ was plotted. All the measured power values $P(\varphi)$ were rated to the value $P\left(0^{\circ}\right)$ that is the measured power in a point located on the line normal on the antenna center at $\varphi=0^{\circ}$.

From Figure 12 one may find that the radiation characteristic in the longitudinal plane is tilted in the forward direction, the maximum radiated power occurring at an angle $\varphi \cong+14^{\circ}$. The radiated power decreases in the backward direction having a secondary radiation lobe at $\varphi \cong$ $-35^{\circ}$.

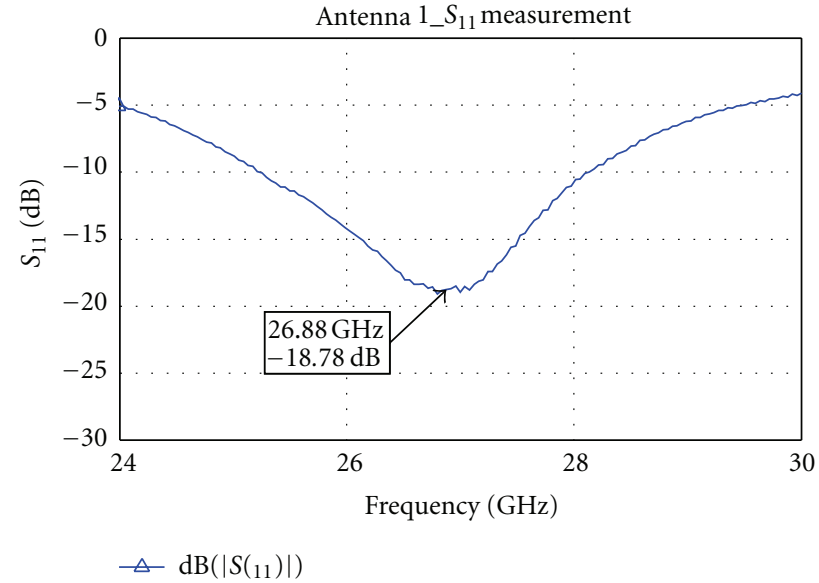

FIGURE 10: Measured return loss of CRLH Antenna \#1 for frequency sweep between $24 \mathrm{GHz}$ and $30 \mathrm{GHz}$.

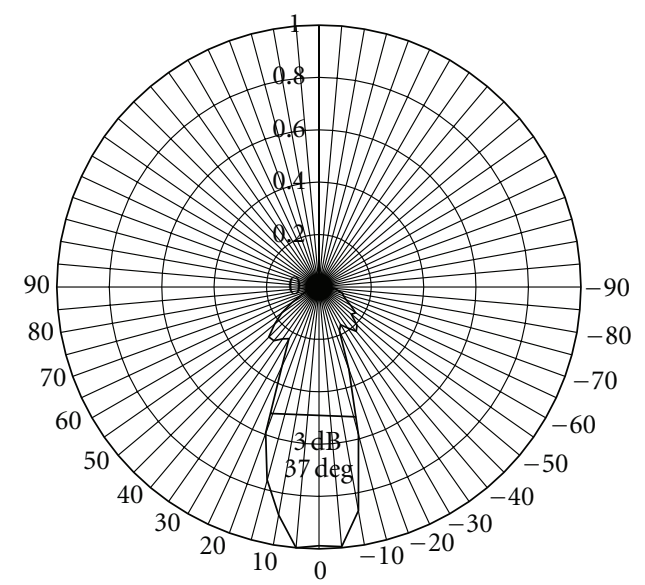

FIgURE 11: Radiation pattern in transversal plane $(\theta)$ of the CRLH Antenna \#1 sample at $f=26.88 \mathrm{GHz}$.

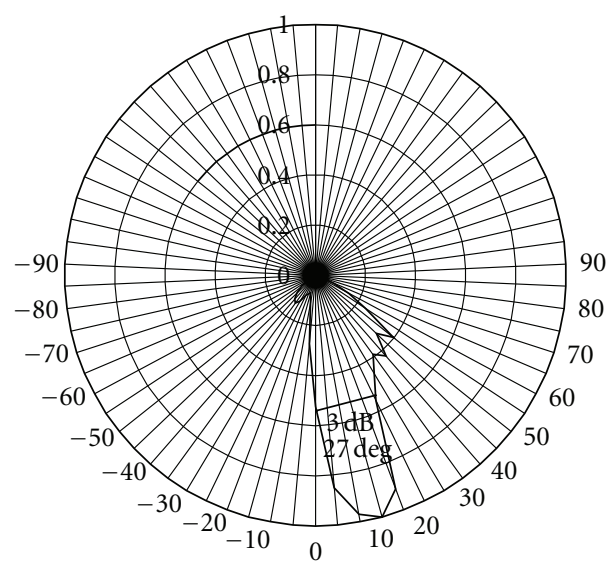

FIgURE 12: Radiation pattern in longitudinal plane $(\varphi)$ of CRLH Antenna \#1 sample at $f=26.88 \mathrm{GHz}$. 


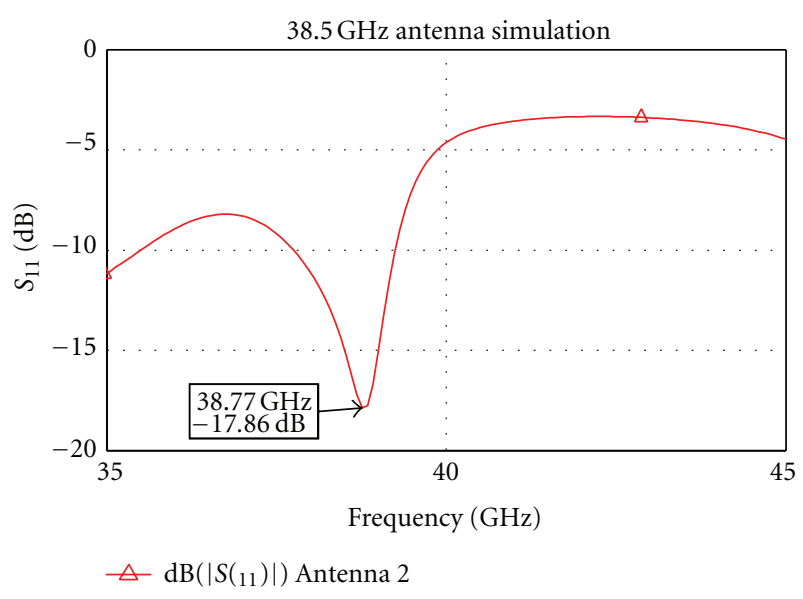

Figure 13: Return loss simulation of the CRLH Antenna \#2 in the $35 \mathrm{GHz}-45 \mathrm{GHz}$ frequency domain.

The CRLH antenna gain was evaluated at $f=27 \mathrm{GHz}$, the measured data being: $\lambda=11.11 \mathrm{~mm}, R=100 \mathrm{~mm}$, power at the emitting antenna: $P_{t}=0.45 \mathrm{~mW}$, power at the receiving antenna $P_{r}=1.29 \mathrm{E}-04 \mathrm{~mW}$. By applying relation (2), the results show $G_{i}=2.82 \mathrm{dBi}$. It is to worth noting that, due to the longitudinal radiation characteristic tilting in the forward direction, the antenna alignment in measuring setup (see Figure 8) was adjusted to make the maximum radiation of both antenna face each other.

4.3. Experimental Results for $38.5 \mathrm{GHz} C R L H$ Antenna. The simulation of reflection loss of the CRLH Antenna \#2 in the $35 \mathrm{GHz}-45 \mathrm{GHz}$ frequency band was made with the CST dedicated software. The simulation presented in Figure 13 indicate as working frequency $f=38.77 \mathrm{GHz}$, where the antenna's return loss is $S_{11}=-17.86 \mathrm{~dB}$.

Measurements of the resonant frequency, return loss, radiation characteristics, and gain were made using the same setups and methods previously presented. The return loss and the resonant frequency were measured on-wafer and the radiation characteristic and the gain, by mounting the antenna structure on specialized test fixture, as in Figure 5(b).

The measured $S_{11}$ in $35 \mathrm{GHz}-45 \mathrm{GHz}$ frequency range is presented in Figure 14.

It may be observed that the return loss is $S_{11}=-38,5 \mathrm{~dB}$ at resonant frequency $38.82 \mathrm{GHz}$ denoting a very good matching of the radiating structures. Besides, values of the $S_{11}$ parameter lower than $-20 \mathrm{~dB}$ are observable in a rather large frequency bandwidth extending from $36.12 \mathrm{GHz}$ to 41.08 GHz.

The radiation characteristics in the transversal $(\theta)$ plane is presented in Figure 15 where the received powers at different angles were normalized to the maximum power value in the domain $\theta \in\left(-90^{\circ}-+90^{\circ}\right)$.

The $-3 \mathrm{~dB}$ beamwidth is approx. $17^{\circ}$ and the side lobes appear at approx. $\pm 50^{\circ}$ with amplitudes lower by $\sim 6 \mathrm{~dB}$ compared to the main lobe.



Figure 14: Return losses $S_{11}$ versus frequency for two CRLH Antenna \#2 sample.

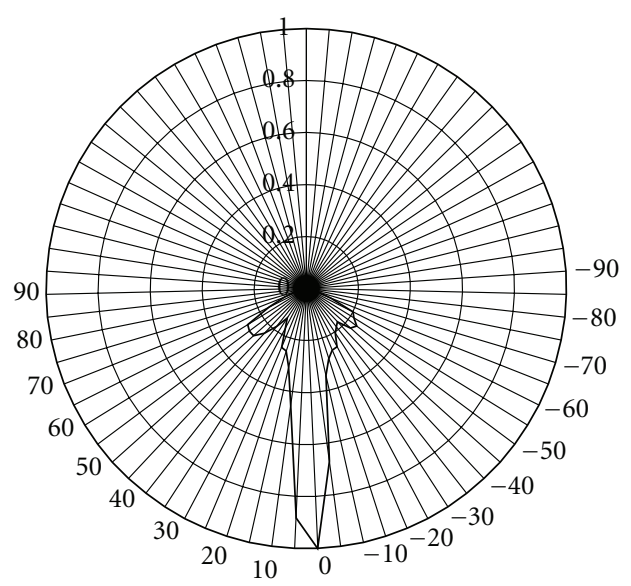

FIGURE 15: Radiation pattern in transversal plane $(\theta)$ for CRLH Antenna \#2 sample at $f=38.82 \mathrm{GHz}$.

Measurements were, also, made at $f=38.8 \mathrm{GHz}$ in the longitudinal plane $(\varphi)$ of the CRLH Antenna \#2 sample. The results are shown in Figure 16 where the radiated power at different angles were plotted. All the received power values were rated to the value $P\left(0^{\circ}\right)$ meaning the measured value of the received power in a point located on the line normal on the antenna center at $\varphi=0^{\circ}$.

Antenna \#2 gain was obtained as previously using the De Friis relation (2), the measurement setup being the same as in Figure 8. The gain was evaluated in the frequency domain $38 \mathrm{GHz}-39 \mathrm{GHz}$. In this frequency domain the gain was $G_{i}=1.08 \mathrm{dBi}$ at $38 \mathrm{GHz}$ and $G_{i}=1.2 \mathrm{dBi}$ at $38.6 \mathrm{GHz}$. The maximum obtained value was $G_{i}=1.75 \mathrm{dBi}$ at $f=$ $38.2 \mathrm{GHz}$ where: $\lambda=7.78 \mathrm{~mm}, R=100 \mathrm{~mm}$, power at the emitting antenna: $P_{t}=0,278 \mathrm{~mW}$, power at the receiving antenna $P_{r}=2.39 E-05 \mathrm{~mW}$. Comparing with the data obtained for Antenna \#1 (27 GHz) the matching is better, the directivity is higher, and the beam-width is smaller. However, the measured gain of Antenna \#2 is smaller than of Antenna \#1 due to the increased losses at higher frequencies in the 


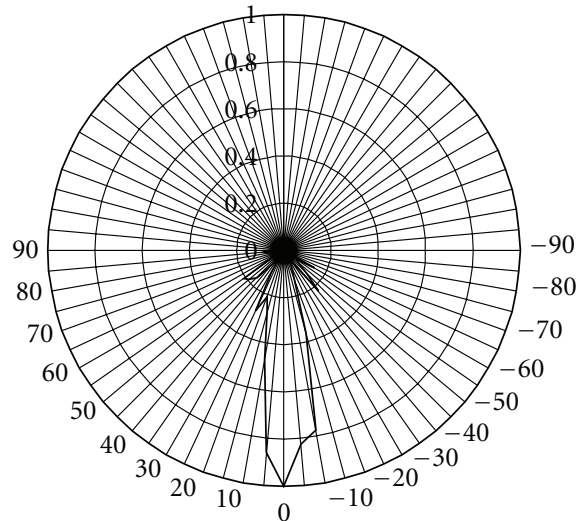

Figure 16: Radiation pattern in longitudinal plane $(\varphi)$ of CRLH Antenna \#2 sample at $f=38.8 \mathrm{GHz}$.

emitting as well as in the receiving antenna on the measuring chain.

\section{Conclusion and Comments}

Two ZOR millimeter wave CRLH CPW antennas on silicon substrate on two different frequencies in the mm-wave domain ( $f=27 \mathrm{GHz}$ and $f=38.5 \mathrm{GHz}$ ) were designed, processed, and measured as part of two integrated circuits.

5.1. Results for the $27 \mathrm{GHz}$ CRLH CPW Antennas. The measured return-loss and resonant frequency for Antenna $\# 1$ sample were: $S_{11}=-18.78 \mathrm{~dB}$ at $f=26.88 \mathrm{GHz}$ demonstrating good agreement with the simulated data. The $3 \mathrm{~dB}$ beam width of radiation lobe is approx. $37^{\circ}$. Concerning the gain, the values computed from the measured data using De Friis relation give $G_{i}=2.82 \mathrm{dBi}$ at $f=27 \mathrm{GHz}$.

5.2. Results for the $38.5 \mathrm{GHz} C R L H C P W$ Antennas. For the $38.5 \mathrm{GHz}$ CRLH Antenna \#2 sample, the measured returnloss was $S_{11}=-38.5 \mathrm{~dB}$ at $f=38.82 \mathrm{GHz}$ showing a good conformity with the simulated data and denoting, also, a very good matching of the radiating structures. Return loss values lower than $-20 \mathrm{~dB}$ are observable in a frequency bandwidth extending from $36.12 \mathrm{GHz}$ to $41.08 \mathrm{GHz}$. The $-3 \mathrm{~dB}$ beam width of radiation lobe is approx. $17^{\circ}$.

The gain in the frequency domain is $G_{i}=1.08 \mathrm{dBi}$ at $f=38 \mathrm{GHz}$ and $G_{i}=1.2 \mathrm{dBi}$ at $f=38.6 \mathrm{GHz}$. Also, the gain is approx. constant in the $38 \mathrm{GHz}-38.6 \mathrm{GHz}$ frequency domain. A maximum gain value of $G_{i}=1.75 \mathrm{dBi}$ was obtained at $f=38.2 \mathrm{GHz}$.

For both the CRLH antenna samples there is a small frequency difference between the computed resonant frequency and the measured one for the best return loss in the working frequency band. These differences are due to the metallic layer over etching in the device processing step. These differences were eliminated in a second technological run by appropriately changing the mask layout. Also, some differences occur due to the fact that the return loss and resonant frequency of CRLH antenna structures were measured on-wafer and the gain and the radiation characteristic were evaluated with the antenna structures mounted on the test fixture. The mechanics and the antenna structure contacting to the test fixture connector generate this slight frequency displacement. However, the results to be retained are those obtained from on-wafer measurements because the antenna will work integrated on silicon substrate and not as separately encapsulated device.

\section{References}

[1] V. G. Veselago, "The electrodynamics of substances with simultaneously negative values of e and $\mu$," Soviet Physics Uspekhi, vol. 10, no. 4, p. 509, 1968.

[2] J. B. Pendry, A. J. Holden, D. J. Robbins, and W. J. Stewart, "Low frequency plasmons in thin-wire structures," Journal of Physics Condensed Matter, vol. 10, no. 22, pp. 4785-4809, 1998.

[3] C. Caloz and T. Itoh, "Invited-novel microwave devices and structures based on the transmission line approach of metamaterials," in Proceedings of the IEEE MTT-S International Microwave Symposium Digest, pp. 195-198, Philadelphia, Pa, USA, June 2003.

[4] C. Caloz, T. Itoh, and A. Rennings, "CRLH metamaterial leaky-wave and resonant antennas," IEEE Antennas and Propagation Magazine, vol. 50, no. 5, pp. 25-39, 2008.

[5] A. Sanada, M. Kimura, I. Awai, C. Caloz, and T. Itoh, "A planar zeroth-order resonator antenna using a left-handed transmission line," in Proceedings of the 34th Conference on European Microwave Conference, pp. 1341-1344, Amsterdam, The Netherlands, October 2004.

[6] S. Simion, R. Marcelli, and G. Sajin, "Small-size CPW silicon resonating antenna based on transmission-line meta-material approach," Electronics Letters, vol. 43, no. 17, pp. 908-909, 2007.

[7] S. Simion, G. Sajin, R. Marcelli, and F. Craciunoiu, "Silicon resonating antenna based on CPW composite Right/LeftHanded transmission line," in Proceedings of the 37th European Microwave Conference (EUMC '07), pp. 478-481, Munchen, Germany, October 2007.

[8] R. Van Dijk, A. Neto, J. A. G. Akkermans, and J. Mills, "EBG-based $60 \mathrm{GHz}$ on-chip antenna in passive silicon," in Proceedings of the 38th European Microwave Conference (EuMC '08), pp. 682-685, Amsterdam, The Netherlands, October 2008.

[9] C. C. Yu, M. H. Huang, L. K. Lin, and Y. T. Chang, "A compact antenna based on metamaterial for WiMAX," in Proceedings of the Asia Pacific Microwave Conference (APMC '08), Hong Kong, December 2008, Paper J2-05.

[10] P. Seongmin, B. Jung-Woo, C. Sang-Hyeok, and K. Young-Sik, "A metamaterial-based symmetrical periodic antenna with efficiency enhancement," in Proceedings of the Asia Pacific Microwave Conference (APMC '08), Hong Kong, December 2008, Paper A3-49.

[11] R. W. Ziolkowski, P. Jin, and C. Lin, "Electrically small metamaterial-inspired antennas: the next generation," in Proceedings of the 3rd International Congress on Advanced Electromagnetic Materials in Microwaves and Optics, pp. 44446, London, UK, August 2009.

[12] A. A. Basharin and N. P. Balabha, "The radiation of antennas based on metamaterial waveguides," in Proceedings of the 3 rd International Congress on Advanced Electromagnetic Materials in Microwaves and Optics, pp. 224-2226, London, UK, August 2009. 
[13] S. Eggermont, R. Platteborze, and I. Huynen, "Analysis of radiation in a metamaterial leaky wave antenna based on complementary split ring resonator," in Proceedings of the 3rd International Congress on Advanced Electromagnetic Materials in Microwaves and Optics, pp. 102-1104, London, UK, August 2009.

[14] S. Simion, R. Marcelli, G. Bartolucci et al., "Composite Right/Left Handed (CRLH) based devices for microwave applications," in Advanced Microwave and Millimeter Wave Technologies: Semiconductor Devices Circuits and Systems", pp. 89-112, INTECH, Vienna, Austria, 2010.

[15] A. C. Bunea, F. Craciunoiu, and Gh. Sajin, "28 GHz CRLH antenna on silicon substrate," in Proceedings of the 41st European Microwave Conference (EuMC '11), pp. 579-5582, Manchester, UK, October 2011.

[16] G. Sajin, F. Craciunoiu, A. Dinescu, and I. Mocanu, "38 $\mathrm{GHz}$ metamaterial antenna on silicon substrate," in Proceedings of Asia-Pacific Conference on Antennas and Propagation (APCAP '12), Singapore, August 2012.

[17] S. I. Matsuzawa, K. Sato, Y. Inoe, and T. Nomura, "Steerable composite right/left-handed leaky wave antenna for automotive radar applications," in Proceedings of the 36th European Microwave Conference (EuMC '06), pp. 1155-1158, Manchester, UK, September 2006.

[18] K. Mori and T. Itoh, "Distributed amplifier with CRLHtransmission line leaky wave antenna," in Proceedings of the 38th European Microwave Conference (EuMC '08), pp. 686689, Amsterdam, The Netherlands, October 2008.

[19] Takahiro Kawakami and Yasushi Horii, "A compact composite right/left-handed (CRLH) leaky wave antenna composed of 2 $\times 2$ coupled-mushrooms for broadband wireless communications," in Proceedings of the Asia-Pacific Microwave Conference (APMC '11), pp. 674-6677, Melbourne, Australia, December 2011.

[20] K. Mori and T. Itoh, "CRLH metamaterial receiving leaky wave antenna integrated with distributed amplifier," in Proceedings of the Asia Pacific Microwave Conference (APMC '08), Hong Kong, December 2008.

[21] T. Kodera and C. Caloz, "Integrated leaky-wave antenna front-end using a ferrite-loaded open waveguide structure," in Proceedings of the 40th European Microwave Conference (EuMC '10), pp. 469-472, Paris, France, September 2010. 



Submit your manuscripts at

http://www.hindawi.com
